Ardila Linares, I. G., \& Martínez Romero, E. (2021). Propuesta de un sistema de tratamiento de vertimientos en la estación piscícola Propiscol como herramienta de gestión ambiental para la producción pecuaria sostenible. Teknos Revista Científica, 21(1), 34-43.

\title{
Propuesta de un sistema de tratamiento de vertimientos en la estación piscícola Propiscol como herramienta de gestión ambiental para la producción pecuaria sostenible
}

\section{Proposal for a spill treatment system at the Propiscol fish farm as an environmental management tool for sustainable livestock production}

\section{Ivan G. Ardila Linares ${ }^{1}$, Ever Martínez Romero²}

\author{
${ }^{1}$ Docente, Ingeniería Ambiental, Centro de Desarrollo Empresarial - CDE, Universidad Escuela Colombiana de Carreras \\ Industriales, Carrera 19 No. 49 - 20 Bogotá, Colombia. \\ iardilal@ecci.edu.co \\ ${ }^{2}$ Docente Tecnología Agroambiental, Corporación Universitaria Comfacauca, Calle 5 \# 9-50 Santander de Quilichao, \\ Colombia
}

Recibido: 15/feb/2021 Revisado: 30/abr/2021

Aceptado: 30/may/2021 Publicado: 30/jul/2021

\begin{abstract}
Resumen La propuesta de un sistema de tratamiento de aguas residuales para la estación piscícola Propiscol ubicada en el municipio de Santander de Quilichao departamento del Cauca (Colombia) como medida de control para mitigar los impactos ambientales causados por el vertimiento de las aguas servidas productos del proceso de producción de tilapia roja (Oreochromis $s p$ ), se trabajó bajo la metodología desarrollada por la Federación Nacional de Acuicultores de Colombia - FEDEACUA, para la implementación de BPPA - Buenas Prácticas de Producción de la Acuicultura - bajo la Norma Técnica Colombiana NTC 5700, de tal forma que se pueda determinar la línea base para la gestión ambiental. Mediante el uso de metodologías de evaluación de impactos, se identificaron aspectos e impactos, relacionándolos con actividades, valoración e importancia, concluyendo que la actividad que más contamina en la producción de tilapia es el manejo del cultivo con una importancia de 27 puntos, relacionado con el aspecto ambiental de utilización de agroquímicos; seguido del aspecto ambiental: ajuste de la alimentación con una importancia del impacto de 24 puntos. La actividad que menos contamina es aseo de instalaciones, para el aspecto ambiental manejo de residuos sólidos con una importancia del impacto de 12 puntos.
\end{abstract}

Palabras claves Aguas Residuales; Impacto Ambiental; Acuicultura; Gestión Ambiental; Costos.

\begin{abstract}
The proposal of a wastewater treatment system for the Propiscol fish farm located on Santander de Quilichao, department of Cauca (Colombia) as a control measure to mitigate the environmental impacts caused by the discharge of water served products of the production process of red tilapia (Oreochromis sp), we worked under the methodology developed by the National Federation of Aquacultures of Colombia - FEDEACUA, for the implementation of BPPA - Good Production Practices of Aquaculture - under the Colombian Technical Standard NTC 5700, so that the baseline for environmental management can be determined. Through the use of impact assessment methodologies, aspects and impacts were identified, relating them to activities, valuation and importance, concluding that the activity that pollutes the most in tilapia production is the management of the crop with an importance of 27 points, related to the environmental aspect of the use of agrochemicals; followed by the environmental aspect: feeding adjustment with an impact importance of 24 points. The least polluting activity is cleaning facilities, for the environmental aspect solid waste management with an impact importance of 12 points.
\end{abstract}

Keywords Wastewater; Environmental Impact; Aquaculture; Environmental Management; Cost. 


\section{Introducción}

Colombia es un país tropical con gran cantidad de cuencas hidrográficas que lo identifican como un lugar estratégico en recursos hídricos en el mundo; con uno de los más altos índices de biodiversidad del planeta, que incluye una gran cantidad de peces. Cuenta con múltiples sistemas hidrológicos en cuerpos de agua dulce, salobres y marinos, variedad de pisos térmicos con características climáticas estables a lo largo del año, terrenos con aptitud y con una vasta red fluvial que recorre casi todo el país, lo que ofrece un muy amplio potencial para el desarrollo de la acuicultura (AUNAP, 2014, p. 9).

La Acuicultura, al ser considerado como uno de los sectores de producción de alimentos más acelerado mundialmente, representa el $50 \%$ de los productos pesqueros, destinados a la alimentación, por ello se establece relaciones entre los países para el intercambio de información relacionada con la pesca. Esto permite el desarrollo de una gestión responsable en el proceso de la piscicultura y acuicultura, siendo la Organización de las Naciones Unidas para la Agricultura y la Alimentación - FAO, la entidad que lidera este tipo de proyectos en la agroindustria. Razón por la cual el estudio de caso de la piscícola Propiscol debe orientarse, no sólo a las normas y legislación colombiana, sino también a las orientaciones técnicas y ambientales de dicha organización (FAO, 2017, párr. 2).

La propuesta se enfoca en la gestión del uso de recursos y manejo de impactos ambientales generados y evidenciados en los vertimientos de la piscícola. Básicamente está enmarcada en el análisis situacional, revisión bibliográfica, diagnóstico, ensayos de laboratorio, propuesta de alternativas y mejoramiento al sistema de tratamiento de vertimientos, con el fin de llevar un proceso acuícola sostenible y amigable con el medio ambiente, así mismo el resultado de este proyecto puede ser implementado en actividades similares y ser apoyado por el Ministerio de Ambiente y Desarrollo Sostenible, para la contribución al desarrollo del sector agrícola y pecuario.

\section{Antecedentes}

El artículo 286 del Decreto 2811 de 1974 (MADS, 2019, p. 54) por el cual se dicta el Código Nacional de Recursos Naturales Renovables y de Protección al Medio Ambiente, define la acuicultura como el cultivo de organismos hidrobiológicos con técnicas apropiadas, en ambientes naturales o artificiales, y generalmente bajo control.

Según la $\operatorname{AUNAP}(2014$, p. 8) la FAO define la acuicultura como "... el cultivo de organismos acuáticos en áreas continentales o costeras, que implica por un lado la intervención en el proceso de crianza para mejorar la producción y por el otro la propiedad individual o empresarial del stock cultivado". Un año después, en 2014 se redefine como "El cultivo de organismos acuáticos, incluyendo peces, moluscos, crustáceos y plantas acuáticas, que implica la intervención del hombre en el proceso de cría para aumentar la producción, en operaciones como la siembra, la alimentación, la protección de los depredadores, etc. La actividad de cultivo también presupone que los individuos o asociaciones que la ejercen son propietarios de la población bajo cultivo" (párr. 7).

La acuicultura debe enmarcarse en criterios de desarrollo sostenible, como "El manejo y la conservación de la base de recursos naturales y la orientación del cambio tecnológico e institucional, de tal manera que se asegure la continua satisfacción de las necesidades humanas para las generaciones presentes y futuras". Este desarrollo sostenible (en los sectores agrícola, forestal y pesquero) conserva la tierra, el agua y los recursos genéticos vegetales y animales, no degrada el medio ambiente y es técnicamente apropiado, económicamente viable y socialmente aceptable" (FAO, 2014, p. 24).

La Ley 13 de 1990 o Estatuto General de Pesca (MADR, 2019, p. 9) permite clasificar la acuicultura desarrollada en Propiscol de la siguiente manera:

Según el medio, como acuicultura continental, la que se realiza en los ríos, lagos, lagunas, pozos artificiales y otras. 
Según su manejo y cuidado, en acuicultura semi intensiva, cuya siembra es aquella en la que se proporciona alimentación suplementaria, además del alimento natural, con mayor nivel de manejo y acondicionamiento del medio ambiente.

Y según las fases del ciclo de vida, en ciclo completo o cultivo integral; ya que abarca el desarrollo de todas las fases del ciclo de vida de las especies en cultivo.

El país cuenta con tres áreas pesqueras muy amplias y diferentes que son el Océano Pacífico, el Mar Caribe y las áreas continentales, pero los volúmenes de captura en las tres han presentado una tendencia decreciente que se ha acelerado en los últimos años. Los efectos negativos sobre la producción pesquera se dan por diferentes causas como son la colmatación y disminución de la profundidad de los lechos de los ríos ocasionada principalmente por la deforestación en los nacimientos y a lo largo de las riveras de los ríos, lo que impide las adecuadas migraciones de los peces; la desecación de muchos de los cuerpos de agua que conforman las cuencas, lo que disminuye las áreas de larvicultura; la contaminación con metales pesados procedentes de explotaciones mineras, las aguas servidas de los asentamientos humanos ribereños y la pesca de forma inadecuada por realización de capturas en épocas de reproducción, irrespeto de las tallas mínimas de captura establecidas y el uso de artes de pesca no selectivos.

Propiscol pertenece a la acuicultura continental y conforma un área de la piscicultura, constituida con un número relativamente alto de productores localizados en la mayoría de los departamentos de la Región Andina.

\section{Metodología}

La metodología desarrollada por la Federación Nacional de Acuicultores de Colombia - FEDEACUA, para la implementación de BPPA - Buenas Prácticas de Producción de la Acuicultura - bajo la Norma Técnica Colombiana NTC 5700, permite capturar, analizar y evaluar información en campo de tal forma que se pueda determinar la línea base para la gestión de aguas residuales.

\section{a. Alcance temático}

La metodología comprende identificar dos grandes procesos para analizar el manejo y disposición de las aguas residuales para la Unidad Productiva Propiscol (UPP), el macro proceso de producción de la piscícola y el macro proceso de gestión ambiental.

\section{b. Alcance geográfico}

Propiscol es una empresa dedicada a la producción de alevinos y carne de tilapia roja. La estación piscícola aplica nuevas tecnologías al sector para suplir las necesidades de la creciente industria regional y nacional, siendo en mayor rigor: la calidad genética y rendimiento, lo que traduce mayor rendimiento.

La productora se encuentra en ubicada en la vereda Santa María cabecera del municipio de Santander de Quilichao departamento del Cauca (Colombia). Sus coordenadas geográficas corresponden a $3^{\circ} 00^{\prime} 42,9^{\prime \prime} \mathrm{N}$ y $76^{\circ} 30^{\prime} 34.5^{\prime \prime} \mathrm{W}$, delimitando por el norte con los municipios de Villarrica y Jamundí, por el sur con el municipio de Caldono, por el oriente con los municipios de Caloto y Jambaló y al occidente con el municipio de Buenos Aires (FEDEACUA, 2015, p. 7).

\section{c. Captura}

Visita para la captura de información en las instalaciones de la Unidad productiva Propiscol (UPP) donde se utilizaron herramientas como celular, calculadora, información por medio de entrevista, información secundaria, libreta de apuntes y cámara fotográfica. Se recolecta información necesaria para el diagnóstico. Se observa una piscina con polisombra como medida de control de los depredadores.

Para determinar los aspectos e impactos ambientales presentes en la empresa se realizaron visitas a cada una de las áreas de estudio donde se identificó la situación actual con el fin de establecer 
indicadores y evaluación de los respectivos componentes ambientales, mediante una matriz de impactos (Tabla 1) relacionando: Actividades, aspecto, impacto, valoración e importancia.

Tabla 1. Identificación de Aspectos e impactos

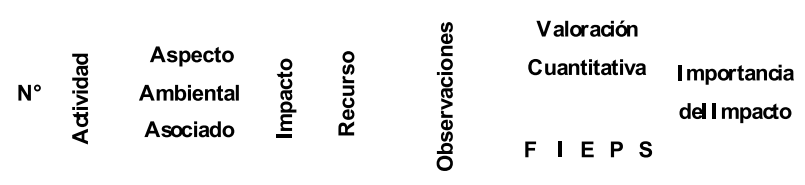

Fuente: Conesa. 1997

El valor de importancia de los impactos ambientales se calcula a través de la expresión:

$$
I M=F(P) * C *(I+E+P+S)
$$

Dónde:

IM = Importancia del Impacto.

$\mathrm{F} \circ \mathrm{P}=$ Frecuencia (utilizada cuando se trata de impactos reales) o Probabilidad (utilizada cuando se trata de impactos potenciales).

$\mathrm{C}=$ Consecuencia.

El valor de la Consecuencia (C) en la fórmula se evaluara a partir de los criterios de:

$\begin{array}{ll}\mathrm{I} & =\text { Intensidad. } \\ \mathrm{E} & =\text { Extensión. } \\ \mathrm{P} & =\text { Persistencia } . \\ \mathrm{S} & =\text { Sensibilidad. }\end{array}$

El resultado de dicha fórmula varía entre un valor mínimo de 4 y un máximo de 36 que clasifica el rango en bajo, medio y alto; tal como se relaciona en la tabla 2.

Tabla 2. Rango de importancia

\begin{tabular}{ccc}
\hline $\begin{array}{c}\text { Rangode Importancia } \\
\text { del Impacto }\end{array}$ & $\begin{array}{c}\text { Rango Significancia } \\
\text { del Aspecto }\end{array}$ & Prioridad \\
\hline $4-11$ & Bajo terciaria \\
$12-16$ & Medio Secundaria \\
$17-36$ & Alto Primaria \\
\hline
\end{tabular}

Fuente: Conesa. 1997
Las tablas 3 y 4 estructuran la medición y el criterio de evaluación del impacto, respectivamente.

El impacto identificado se clasifica en positivo o negativo, según la naturaleza del mismo.

Tabla 3. Medición del Impacto

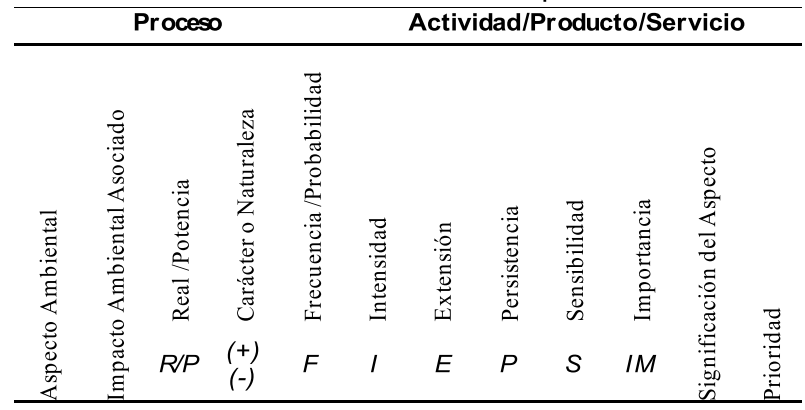

Fuente: Conesa. 1997

Tabla 4. Criterio de evaluación

\begin{tabular}{|c|c|c|c|}
\hline \multirow[b]{2}{*}{ Criterio de Evaluación } & \multicolumn{3}{|r|}{ Escala deEvaluación } \\
\hline & Valor & Clasificación & Expresión \\
\hline & $(+)$ & Positivo & $\begin{array}{c}\text { Representan una mejora en las cualidades intrínsecas de } \\
\text { los componentes del medio físico o social, contribuyendo } \\
\text { a aumentar su complejidad orgánica funcional y su } \\
\text { estabilidad regional. Ejemplo Reutilización del agua, } \\
\text { Reciclaje de los Residuos, entre otros. }\end{array}$ \\
\hline $\begin{array}{l}\text { Naturaleza del Impacto }(\mathrm{N}) \text { : } \\
\text { Se refiere al efecto beneficioso } \\
(+) \text { o perjudicial }(-) \text { de los } \\
\text { diferentes aspectos ambientales } \\
\text { que van a incidir sobre los } \\
\text { componentes considerados. }\end{array}$ & $(-)$ & Negativo & $\begin{array}{l}\text { Suponen un empeoramiento de las condiciones naturales } \\
\text { o sociales del Medio Ambiente favoreciendo su } \\
\text { desestabilización y conduciéndolo hasta una mayor } \\
\text { simplicidad funcional concretada en una disminución de } \\
\text { la riqueza biológica y de las relaciones ecológicas de } \\
\text { autorregulación. Ejemplo: Consumo de Recursos, } \\
\text { generación y descarga de residuos, emisiones de gases, } \\
\text { vertimiento de aguas residuales, situaciones potenciales } \\
\text { de fugas derrames, incendios, explosiones, entre otros. }\end{array}$ \\
\hline
\end{tabular}

Fuente: Conesa. 1997

\section{Diagnostico}

En la UPP se realiza la mejora de poblaciones contando con un trabajo de cría selectiva, que procura mantener la aptitud genética, consiguiendo los procedimientos adecuados para la selección de reproductores y producción de huevos, larvas y alevinos. Tal cual, como lo define el estatuto general de pesca reglamentado por la Ley 13 de 1990, la unidad se puede clasificar como acuicultura continental realizada en pozos artificiales, siendo una explotación semi-intensiva con un ciclo de vida completo o cultivo integral. Luego de identificar los procesos productivos de la actividad como lo son: el 
proceso de reproducción y alevinaje, la siembra, el procedimiento de reversión sexual, seguimiento en cultivo (cosecha) mediante análisis de la producción mensual y anual, despacho, limpieza y desinfección; se caracterizan los parámetros fisicoquímicos de la tabla 5.

Tabla 5. Parámetros fisicoquímicos del agua para especies de clima cálido

\begin{tabular}{cc}
\hline PARÁM ETROS & RANGOS \\
\hline Temperatura & $25-32{ }^{\circ} \mathrm{C}$ \\
\hline Oxígeno disuelto & $>5 \mathrm{mg} / \mathrm{L}$ \\
\hline $\mathrm{pH}$ & $50-150 \mathrm{mg} / \mathrm{L}$ \\
\hline Alcalinidad Total & $80-110 \mathrm{mg} / \mathrm{L}$ \\
\hline Dureza Total & $60-120 \mathrm{mg} / \mathrm{L}$ \\
\hline Calcio & $0.1 \mathrm{mg} / \mathrm{L}$ \\
\hline Nitritos & $1.5-2 \mathrm{mg} / \mathrm{L}$ \\
\hline Nitratos & $0.1 \mathrm{mg} / \mathrm{L}$ \\
\hline Amonio Total & $0.05-0.2 \mathrm{mg} / \mathrm{L}$ \\
\hline Hierro & $0.15-0.2 \mathrm{mg} / \mathrm{L}$ \\
\hline Fosfatos & $5-10 \mathrm{mg} / \mathrm{L}$ \\
\hline Dióxido de carbono & $0.01 \mathrm{mg} / \mathrm{L}$ \\
\hline Sulfuro de hidrógeno & 2018
\end{tabular}

Fuente: Propiscol. 2018

En cuanto a la Gestión del Recurso Hídrico, el programa para la gestión del agua residual del cultivo, incluye todas las acciones encaminadas a prevenir, gestionar, compensar o corregir los riesgos generados por los residuos líquidos producidos en y por el cultivo.

Propiscol no cuenta con el sistema de tratamiento, ni registro ni permiso de vertimiento o de reúso del agua residual tratada exigido por la Autoridad ambiental competente, de acuerdo con lo dispuesto en el Decreto 1076 de 2015, Decreto 050 de 2018 y la Resolución MADS 1207 de 2014. De este trámite legal se derivan los planes y programas que la empresa debe tener en cuenta en la gestión del recurso hídrico.

Adicionalmente, se encontró que no hay un programa de monitoreo y caracterización de efluentes que permitan identificar los valores permitidos de los parámetros fisicoquímicos y microbiológicos, establecidos en la Resolución 0631 de 2015, artículo
15. En la revisión de la normativa se evidencia que hay ausencia de ordenamiento jurídico que hace que la matriz legal éste incompleta.

Donde debería contar con un sistema de tratamiento, registro y permiso de vertimientos, los cuales permitirán identificar, evaluar y controlar los impactos que genera la descarga del agua de piscicultura al agua superficial. Adicional, el proceso y el trámite ambiental es un mecanismo que evita sanciones y da cumplimiento de la normatividad ambiental vigente.

\section{Resultados y análisis}

De acuerdo a las visitas realizadas a la estación piscícola Propiscol, a los documentos suministrados por la empresa, las observaciones realizadas en la fase de campo y la información suministrada por los colaboradores, junto con el análisis del cumplimiento normativo encontrado en la fase de diagnóstico, se puede analizar el estado del agua antes de la captación y durante el proceso de producción para determinar los cambios substanciales y comprender el proceso que tiene el agua al interior de la actividad económica, esto, con el fin de proponer el tratamiento antes del vertimiento.

Los datos recolectados en campo durante 4 meses en 10 de las unidades productivas, evidencian el estado del agua en los estanques en parámetros fisicoquímicos como el oxígeno disuelto, la temperatura y el $\mathrm{pH}$. La tabla 6 muestra el promedio de 100 datos por unidad productiva y el promedio general de los parámetros in situ presentes en el proceso productivo.

El promedio de la temperatura de los estanques presenta una desviación estándar de $0,56{ }^{\circ} \mathrm{C}$ y es acorde a la temperatura ambiente promedio de la zona. Bain \& Stevenson (1999, p. 23) define una dependencia de la concentración de oxígeno disuelto respecto a la temperatura del agua con consecuencias a los ecosistemas acuáticos desde la anoxia hasta la sobresaturación. Los datos registrados corresponden a porcentaje de saturación, más no de concentración. Éste parámetro es sin duda uno de los indicadores 
más importantes de la calidad del agua, normalmente los valores varían entre los 7.0 y $8.0 \mathrm{mg} / \mathrm{L}$ siendo la fuente principal el aire; el cual se difunde rápidamente en el agua por la turbulencia en los ríos o por el viento, como el agua está estancada, ésta es una de las razones de una saturación encontrada en Propiscol: Aceptable e incluso crítica (Pérez, 2003, p 32).

(A)Temperatura $-{ }^{\circ} \mathrm{C}$, (B) Unidades de $\mathrm{pH},(\mathrm{C})$ Porcentaje de saturación.

Tabla 6. Parámetros in situ estación piscícola

\begin{tabular}{cccc}
\hline $\begin{array}{c}\text { ESTANQUE } \\
\mathbf{N}^{\circ}\end{array}$ & $\begin{array}{c}\text { TEMPERATURA } \\
{ }^{\circ} \mathbf{C}\end{array}$ & pH & $\begin{array}{c}\text { SATURACIÓN } \\
\mathbf{\%}\end{array}$ \\
\hline 3 & 24,6 & 7,4 & 51,0 \\
4 & 24,6 & 6,9 & 34,2 \\
5 & 25,9 & 7,3 & 53,1 \\
6 & 24,2 & 8,3 & 68,4 \\
7 & 24,8 & 7,4 & 51,3 \\
8 & 25,3 & 7,2 & 68,4 \\
10 & 25,3 & 7,5 & 68,4 \\
11 & 25,4 & 7,5 & 68,4 \\
13 & 24,8 & 7,6 & 51,0 \\
14 & 25,7 & 7,4 & 51,3 \\
Promedio & $\mathbf{2 5 , 1}$ & $\mathbf{7 , 4}$ & $\mathbf{5 6 , 5}$ \\
\hline
\end{tabular}

Fuente: Propiscol. 2018

La figura 1 (c) muestra las condiciones aceptables de saturación de oxígeno en 4 estanques, (b) un promedio de 7,4 unidades de $\mathrm{pH}$ en el agua del proceso productivo y (a) una temperatura promedio de $25,1^{\circ} \mathrm{C}$

Respecto al proceso productivo de la estación, la tabla 7 muestra el agua para abastecer la capacidad instalada equivalente a las 10 unidades productivas, que son de aproximadamente $2.000 \mathrm{~m}^{3}$ según la etapa del proceso y con base en el tamaño de las piscinas.

Adicional al estudio técnico se realizó un análisis estratégico mediante la herramienta metodológica DOFA (Matriz de debilidades, oportunidades, fortalezas y amenazas) para la evaluación de las características fuertes y débiles de la estación, con las amenazas y oportunidades externas, pues la propuesta estratégica debe lograr el encaje entre las capacidades internas y la posición competitiva externa.

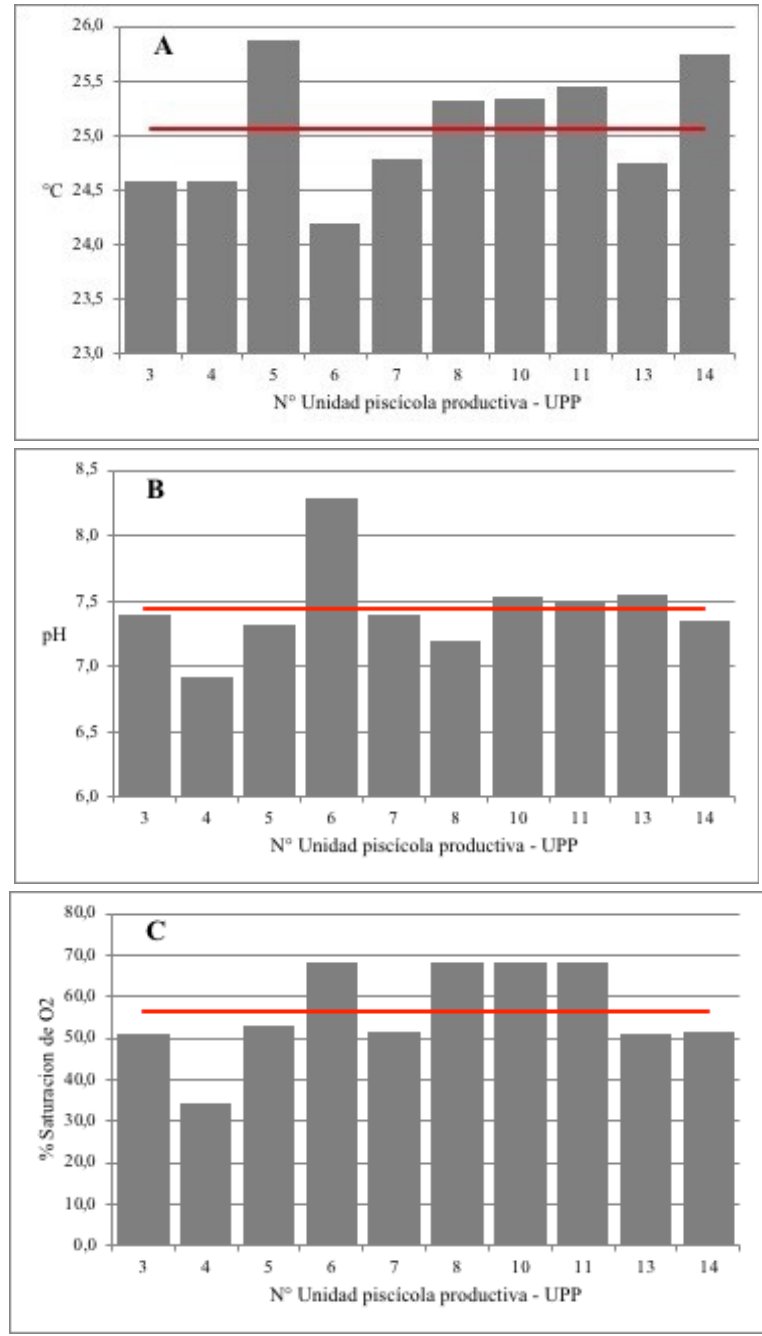

Figura 1 Parámetros físico-químico in situ de los estanques

Primero se identificaron cuáles son los grupos de interés, luego los factores de entorno que pueden afectar la situación y decisión, desde el punto de vista legal, ambiental, tecnológico y financiero. Seguido del análisis DOFA se realiza una matriz de confrontación de donde resulta la propuesta de gestión ambiental.

Tabla 7. Volumen de agua proceso productivo

\begin{tabular}{lc}
\hline \multicolumn{2}{c}{ Dimensiones de lasUPP } \\
\hline Largo & $20,0 \mathrm{~m}$ \\
Ancho & $8,0 \mathrm{~m}$ \\
Profundo & $1,20 \mathrm{~m}$ \\
Volumen por unidad & $192 \mathrm{~m}^{3}$ \\
Cantidad de estanques & 10 \\
Volumen total & $\mathbf{1 . 9 2 0 \mathbf { ~ m } ^ { 3 }}$ \\
\hline
\end{tabular}




\section{Propuesta de Gestión Ambiental y discusión}

Como alternativa de solución frente al desconocimiento de las normas sobre vertimientos para la actividad de piscicultura por parte de Propiscol, se debe aprovechar la micro cuenca de la quebrada El Tajo, que no presenta índices de contaminación, así potencializa la productividad de la actividad acuícola. Igualmente se debe aprovechar la disponibilidad de profesionales en temas ambientales para minimizar las amenazas, como, el cambio climático y aprovechar las buenas prácticas acuícolas, todo esto mediante el diseño de un sistema de tratamiento aguas residuales.

\section{a. Estudio legal}

Para el desarrollo de cualquier actividad que genere impactos al ambiente, es preciso determinar la normatividad vigente aplicable teniendo en cuenta el marco legal respecto del permiso de vertimientos, consistente en el proceso que deben iniciar, solicitar y tramitar las personas naturales o jurídicas que desempeñen actividades 0 presten servicios que generen vertimientos a las aguas superficiales, marinas, o al suelo, previo tratamiento y cumpliendo las normas de vertimiento contempladas en el ámbito nacional colombiano, siendo el decreto 1076 de 2015, el cual ha dispuesto reglas específicas respecto de los vertimientos, conforme a lo dispuesto en el artículo 2.2.3.3.1.3, un vertimiento es la descarga final a un cuerpo de agua, un alcantarillado o al suelo, de elementos, sustancias o compuestos contenidos en un medio líquido, existiendo en consecuencia dos clases: uno puntual que se realiza a partir de un medio de conducción, del cual se puede precisar el punto exacto de descarga al cuerpo de agua, alcantarillado o al suelo; $y$ otro no puntual, o aquel en el que no se puede precisar el punto exacto de descarga al cuerpo de agua $o$ al suelo. La estación piscícola realiza descargas de los vertimientos de manera puntual directamente a un cuerpo de agua superficial, específicamente a la quebrada El Tajo.
En consideración para el cumplimiento del mandato jurídico, Propiscol debe realizar tratamiento antes de realizar el vertimiento.

\section{b. Estudio técnico}

De acuerdo a la caracterización realizada al vertimiento, la demanda bioquímica de oxígeno, los sólidos suspendidos y los sedimentables se encuentran por encima del límite máximo permitido, incumpliendo la reglamentación de vertimientos. En cuanto a grasas y aceites se encuentra muy cerca del estándar.

Una revisión de la literatura permite evidenciar que los procesos biológicos de filtración son los más eficientes, como los humedales artificiales. La figura 2 resume el tratamiento.
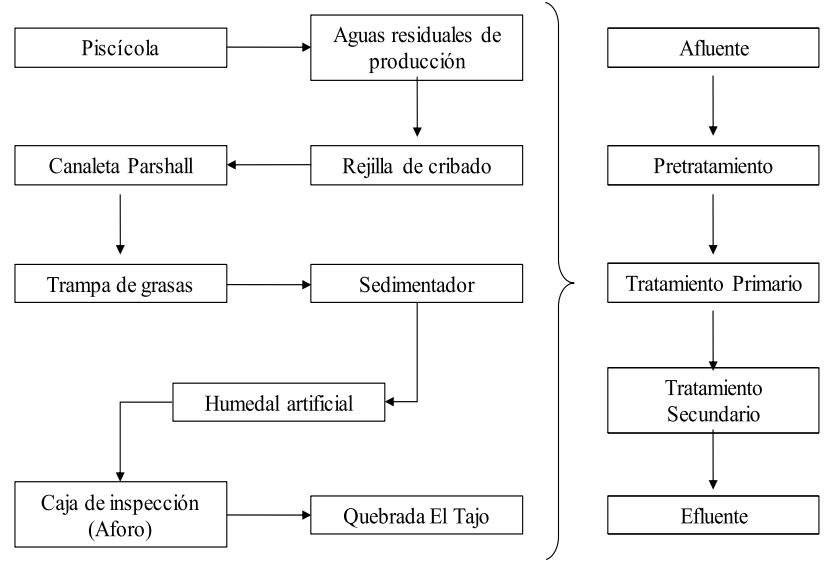

Figura 2 Diagrama de flujo tratamiento

\section{c. Estudio ambiental}

Según la matriz de aspectos e impactos se evidencia que la actividad que más contamina es el manejo del cultivo con una importancia del impacto de 27 puntos referente el aspecto ambiental utilización de agroquímicos; seguido del aspecto ambiental ajuste de la alimentación una importancia del impacto de 24 puntos; la actividad que menos contamina es aseo de instalaciones en lo referente al aspecto ambiental manejo de residuos sólidos con una importancia del impacto de 12 puntos. 


\section{d. Estudio financiero}

Describir y organizar la información monetaria mediante cuadros analíticos y datos adicionales para la evaluación financiera del proyecto ayudan a determinar la rentabilidad además de verificar si existen errores que permitan identificar la falta de gestión, en último, la aprobación o rechazo del proyecto (Sapag, 2008. p. 20).

Sin el proyecto del sistema de tratamiento el VAN (Valor actual neto) es equivalente a $\$ 18.057 .948$ pesos con una tasa interna de retorno del $32,5 \%$ (TIR) y una relación costo beneficio de 1,50 . Esto permite inferir que la piscícola es rentable financieramente.

Ahora bien, como los vertimientos se consideran una externalidad de la piscícola, los costos ambientales se requieren internalizar mediante el sistema de tratamiento. La maquinaria de reemplazo no se tiene en cuenta en el diseño del tratamiento pero se requiere para aumentar la capacidad de la motobomba existente.

Las inversiones iníciales equivalen a cerca de $\$ 23.000 .000$ de pesos, y unas inversiones futuras de $\$ 4.550 .000$ por maquinaria de reemplazo por desgaste. Se contempla una vida útil de las obras de 20 años y de los equipos de 10 , con un caudal promedio de 5 litros por segundo, en caso que aumente el caudal o la concesión cambie, el desgaste será mayor, por ende la vida útil disminuye.

Para ver la variabilidad de la rentabilidad de la piscícola con la implementación del sistema de tratamiento, se incorporan los costos ambientales.

La rentabilidad cambia, disminuyendo en 6,5 puntos porcentuales la tasa interna de retorno y en una tercera parte la relación costo-beneficio, llegando a ser 1. EI VAN por su parte pasa de $\$ 18.057 .948$ a $\$ 13.810 .437$ pesos.

Con los datos obtenidos inicialmente el proyecto financieramente no es atractivo. Sin embargo, algunos análisis como costo-beneficio ambiental son notoriamente significativos, ya que la inversión de 25 millones de pesos evitaría multas diarias de hasta por cinco mil (5.000 SMMLV) salarios mínimos mensuales legales vigentes, el cierre temporal o definitivo de la piscícola o la revocatoria de autorizaciones, concesiones, permisos o registros, sumado a las perdidas sociales y económicas. Sin embargo, esto hace parte de un análisis de valoración económica ambiental de los impactos de la empresa.

Por su parte, un escudo fiscal para favorecer la empresa y el sistema de tratamiento, se puede concebir mediante el manejo de un préstamo.

Al generarse un escudo fiscal, los impuestos bajan $y$ se ve reflejado en el VAN que asciende a $\$ 18.131 .269$ pesos y la relación costo-beneficio aumenta a 1,32; es decir por cada peso invertido se reciben 0,32 . Los intereses pagados reducen la utilidad bruta.

El sistema de tratamiento de aguas residuales, la empresa obtiene un beneficio de 0,50 por cada peso invertido, con el sistema la relación es 1:1, pero con el proyecto financiado mediante un préstamo bancario la relación cambia favorablemente a 1,32. Lo mismo ocurre con el VAN.

\section{Conclusiones}

A partir de la normativa se propuso un sistema de tratamiento de aguas residuales para la estación piscícola Propiscol como medida de control para mitigar los impactos ambientales.

El diagnóstico realizado evidencia que se cuenta con los permisos legales de uso del suelo, captación de aguas superficiales, pero no cuenta con el permiso de vertimientos de aguas residuales tratadas como lo dispone los decretos 1076 de 2015 y 050 de 2018, y la Resolución MADS 1207 de 2014.

Mediante el análisis físico - químico y microbiológico de los vertimientos se caracterizó el agua residual y se comparó con la normativa, de esta forma se propone técnicamente un sistema de tratamiento.

La revisión de la literatura permitió referir estudios de caso exitosos de tratamientos similares, así se escogió la mejor alternativa que cumpliera técnica y legalmente los requisitos ambientales para el normal funcionamiento de la actividad productiva.

La identificación y evaluación de aspectos e impactos ambientales muestra que la actividad que 
más contamina el recurso agua es el manejo del cultivo y la actividad que menos contamina es la de Aseo de las instalaciones.

El estudio ambiental permitió identificar los impactos negativos hacia el recurso hídrico, sumado al incumplimiento legal y posibles sanciones al no tener un sistema de tratamiento de las aguas residuales.

De acuerdo al análisis FODA realizado, al tener numerosos recursos hídricos no contaminados como fortaleza, se deberán establecer lineamientos de desarrollo acuícola a corto, mediano y largo plazo, lo que permitirá tener un impacto socioeconómico positivo.

El diagnóstico y la metodología son elementos claves para la consultoría ambiental. Se debe conocer el problema para proponer soluciones efectivas.

Los indicadores financieros muestran un mayor beneficio apalancando el proyecto con un préstamo para generar un escudo fiscal. Financieramente no es atractiva la inversión; sin embargo, legal, social y ambientalmente es viable, sobre todo sí se cuantifica mediante una valoración económica ambiental.

La relación costo - sanción es positiva, en el entendido que la inversión es menor a las multas por incumplimiento, procesos administrativos $y$ sancionatorios, y demás tramites a los que está expuesta la piscícola.

Agradecimientos. A docentes e investigadores, por los aportes y valiosos comentarios. A las autoridades locales y a la empresa por hacer parte de la construcción de una industria más sustentable y producción alimentaria más limpia.

\section{Referencias}

Alianza El Agro Exporta, (2018a). Departamento del Cauca - Red de Proveedores recuperado de http://www.fedeacua.org/wpcontent/uploads/2018/10/A25_red-de-proveedorescauca.pdf

Alianza El Agro Exporta, (2018b). Evaluación de criterios de cumplimiento frente a la norma técnica colombiana NTC 5700. Buenas Prácticas de Producción de la Acuicultura - Convenio ATGII044-017
AUNAP (2014). Plan Nacional para el Desarrollo de la Acuicultura Sostenible en Colombia - PlaNDAS. Autoridad Nacional de Acuicultura y Pesca. Bogotá, D.C. Colombia

Bohórquez G., L. C., (2015).La importancia del plan de manejo ambiental para la formulación de estrategias de aprovechamiento industrial y económico de los residuos de la cadena piscícola. Universidad Militar Nueva Granada facultad de Ingeniería. Bogotá D.C. Colombia.

Boyd, C. E. (2003). Guidelines for aquaculture effluent management at the farm-level. Aquaculture, 226(1-4), 101-112.

Burbano Gallardo, E. (2018). Influencia de cultivos piscícolas en la calidad del agua y comunidades bacterianas en el sedimento del lago Guamuez. Doctoral dissertation, Universidad Nacional de Colombia-Sede Palmira, Colombia.

Conesa Fernández, V. (1997) Guía Metodológica para la Evaluación de Impacto Ambiental (2da Edición). Editorial Mundi-Prensa, Madrid.

Corantioquia, (2016). Manual de Producción y Consumo Sostenible Gestión del Recurso Hídrico. Piscícolas Cultivo de Trucha y Tilapia.

Corporación Autónoma Regional del Centro de Antioquia. Medellín, Colombia.

CRC, (2010). Plan de Ordenación y Manejo de la Subcuenca Hidrográfica del Río Quinamayo. POMCH Quinamayo. Fases de aprestamiento y diagnóstico. Corporación Autónoma Regional del Cauca. Santander de Quilichao. Colombia.

CRC, (2017). Resolución .Corporación Autónoma Regional del Cauca. Colombia.

Daza, S. J., (2011).Gestión ambiental para sistemas productivos piscícolas, en ecosistemas alto andinos en el contexto de la producción más limpia. Revista Ambiente y Sostenibilidad (1): 18-24 Revista del Doctorado Interinstitucional en Ciencias -Ambientales. Universidad del Cauca - Colombia.

Fedeacua, (2015). Aquicultores. Revista de la Federación Colombiana de Acuicultores. No. 01. Septiembre/octubre del 2015. Federación Colombiana de Acuicultores. Bogotá, D.C., Colombia.

Food and Agriculture Organization. (2003). La ordenación pesquera: El enfoque de ecosistemas en la pesca. FAO Orientaciones Técnicas para la Pesca Responsable, 4(s2).

Food and Agriculture Organization. (2014). La ordenación pesquera: El enfoque de ecosistemas en la pesca. FAO 
Ardila Linares, I. G., \& Martínez Romero, E. (2021). Propuesta de un sistema de tratamiento de vertimientos en la estación piscícola Propiscol como herramienta de gestión ambiental para la producción pecuaria sostenible. Teknos Revista Científica, 21(1), 34-43.

Orientaciones Técnicas para la Pesca Responsable, 4(s2).

Food and Agriculture Organization. (2017). La ordenación pesquera: El enfoque de ecosistemas en la pesca. FAO Orientaciones Técnicas para la Pesca Responsable, $4(\mathrm{~s} 2)$.

García Osorio, G. E. (2018). Evaluación de las características fisicoquímicas del agua en la piscícola de Asojuncal-Huila, asociados al ciclo de producción de la Tilapia roja. Universidad Nacional Abierta y a Distancia. Escuela de Ciencias Agrícolas, Pecuarias y de Medio Ambiente. Neiva, Colombia.

González Acosta, J. A., (2012). Uso y manejo de sedimentos provenientes de piscicultura como base para el manejo sostenible: revisión del tema. Universidad de La Salle. Bogotá, D. C. Colombia.

Henao, A. J. \& Gómez-Rey, A. (2018). De la complejidad jurídica de los vertimientos. Revista Prolegómenos Derechos y Valores, 21, 41, 25-41. DOI: http://dx.doi.org/10.18359/prole.3328

IDEAM, (2013). Zonificación y codificación de unidades hidrográficas e hidrogeológicas de Colombia. Publicación aprobada por el Comité de Comunicaciones y Publicaciones del IDEAM. Bogotá, D. C., Colombia.

Incoder, (2006).Guía Práctica de la piscicultura en Colombia. Instituto Colombiano de Desarrollo Rural, subgerencia de pesca y acuicultura. Bogotá, D.C., Colombia.
Luna Imbacuán, M. A., Campos Bermúdez, F., \& MedinaGutiérrez, O. (2016). Evaluación de las aguas residuales del lavado de estanques multipropósito con cultivo de trucha arcoíris (Oncorhynchus mykiss sp.). Ciencia y Tecnología Agropecuaria, 17(2), 191-202.

MADR (2014).Ministerio de agricultura y desarrollo rural. Autoridad Nacional de Acuicultura y pesca. Plan nacional para el desarrollo de la acuicultura sostenible en Colombia - PlaNDAS. Bogotá, D. C. Colombia.

Pérez, G. R. (2003). Bioindicación de la calidad del agua en Colombia: Propuesta para el uso del método BMWP Col. Universidad de Antioquia.

Sapag Chain, N., \& Sapag Chain, R. (2008). Preparación y evaluación de proyectos. McGraw Hill.

Seoánez, C. M. (1999). Aguas Residuales: Tratamiento por humedales artificiales. Fundamentos científicos. Tecnologías. Diseño. Madrid: Ediciones mundi-prensa

Sotelo Ruíz, C. (2018). Formulación del plan estratégico para el mejoramiento organizativo de Apropesca 2017 2020. Doctoral dissertation. Silvia, Cauca.

Villamizar Ortiz, N. J. (2015). Elaboración de la guía ambiental para el subsector acuicultor en Colombia.

World Health Organization, (2006). Guidelines for the safe use of wastewater, excreta and greywater (Vol. 1). World Health Organization. 